\title{
Modélisation de la couche limite de fond générée par un écoulement de houle
}

\section{Rafik Absi}

Doctorant, Laboratoire de Mécanique, Université de Caen.

Michel Bélorgey

Professeur, Laboratoire de Mécanique, Université de Caen. Groupe Mécanique des Fluides et Génie Côtier.

\section{Résumé}

Nous avons utilisé une technique simple pour simuler numériquement la couche limite générée par un écoulement de houle sur un fond lisse. Les équations de Navier-Stokes, sous une formulation vitesse-pression, sont résolues par un schéma de volumes finis. La validité de la méthode a été testée par comparaison avec des valeurs expérimentales obtenues par Vélocimétrie Doppler Laser en canal à houle. L'étude comparative a permis d'entreprendre une discussion non seulement sur la méthode de résolution et les résultats numériques, mais également une analyse fine de la technique de mesures et des valeurs expérimentales.

\section{Introduction}

La couche limite a un rôle important dans le processus du transport sédimentaire. L'entraînement, la suspension, la déposition et la remise en suspension des particules, sont largement dépendants d'une description correcte des processus se produisant au niveau de cette couche limite.

Pour fournir les gradients verticaux de vitesses qui influencent la formation et le déplacement de sédiments, nous avons choisi un modèle bidimensionnel vertical (2DV) pour réaliser une étude numérique de la couche limite de fond. En effet, les modeles monodimensionnels et bidimensionnels horizontaux ne permettent pas de fournir ces gradients de vitesses, et les modèles tridimensionnels sont coûteux et présentent des problèmes numériques. De plus le modèle 2DV s'adapte bien aux études en canal à houle.

L'étude générale étant complexe, nous devons dans un premier temps modéliser l'écoulement dans la couche limite générée par la houle sur fond lisse. Nous présentons ici nos résultats comparés aux études expérimentales [11] déjà réalisées au laboratoire. Nous présentons en plus une analyse de la méthode numérique d'une part et de la technique expérimentale d'autre part, qui est detaillée dans [2] et [5]. 


\section{Nature de la couche limite}

La couche limite générée par la houle sur un fond marin est généralement turbulente pour diverses raisons, d'abord le grand nombre de Reynolds, puis la nature du fond qui est loin d'être lisse et rarement régulier.

Pour conserver les critères de turbulence, la plupart des études expérimentales ont été réalisées en tunnels oscillants (permettant ainsi d'accéder à de grands nombres de Reynolds) $[6,10]$. Cependant, l'écoulement ainsi réalisé ne correspond pas exactement à un écoulement de houle, au sein duquel nous avons une surface libre, des variations de pression et des champs de vitesses différents. De ce fait, même si les conditions de similitude de Reynolds ne sont pas respectées, il est judicieux de compléter les études précédentes par des études en canal à houle respectant les conditions de Froude. Le problème de la nature de la couche limite, turbulente ou non, reste cependant posé.

Les mesures effectuées par Tcheukam-Toko (1997) en canal à houle pâr Vélocimétrie Doppler Laser ont montré que pour les conditions expérimentales des tests $\mathrm{H} 2$ et $\mathrm{H} 3$, le taux de turbulence dans l'écoulement hors de la couche limite est faible et que les fluctuations des vitesses horizontales très près du fond dans les phases de sommet et de creux de la houle sont non négligeables. Ceci implique que la nature de cette couche limite n'est pas bien définie, car si pour les phases de sommet et de creux on peut éventuellement parler de turbulence, il n'en est plus de même pour les autres phases de la houle. Il a été constaté qu'on est en présence d'un phénomène transitoire soumis à des instabilités excitées par la houle. Selon les critères de Sleath, la couche limite en canal à houle présente un caractère turbulent pour certaines phases de celle-ci. Par contre la même étude de Tcheukam-Toko (1997) montre que pour ces conditions de houle, les tensions de Reynolds étaient nulles au voisinage du fond, et qu'elles restent très faibles dans tout le volume d'eau (figure 1). Il semblerait donc d'après ces résultats, que la contrainte de frottement dans toutes ces couches limites soit contrôlée essentiellement par la viscosité et non par la turbulence [11].

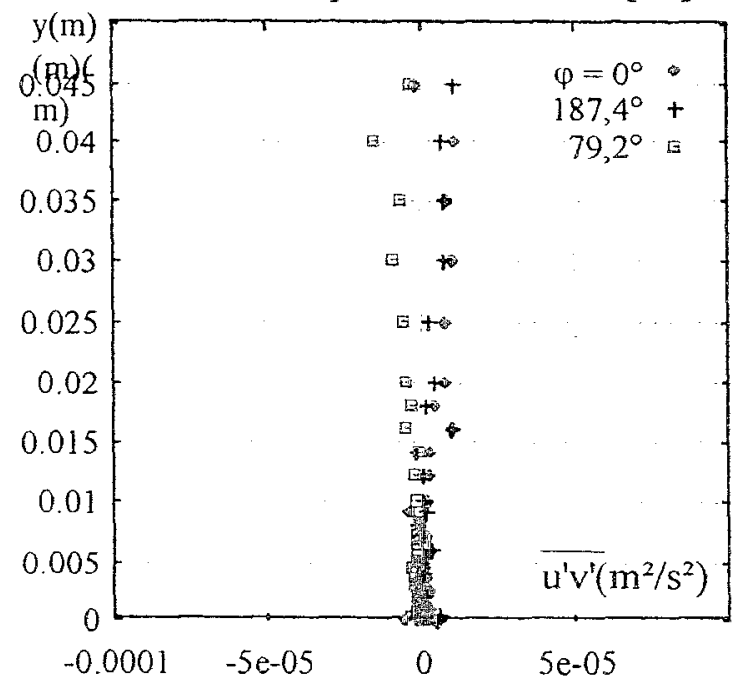

Figure 1. Profil de la covariance de vitesses turbulentes, test H2 (VDL) [11]. 
De ce fait nous avons dans un premier temps exploité l'aspect laminaire de cette couche limite. Néanmoins une analyse de la technique de mesure est effectuée dans la partie 5. Bien entendu, l'introduction d'un modèle de turbulence est envisageable d'abord pour traiter le fond irrégulier par exemple constitué de rides, et pour fournir la diffusivité turbulente, qui permettra de modéliser le transport sédimentaire.

\section{Modélisation Numérique}

\subsection{Description du problème physique}

La simulation d'un écoulement de houle dans un domaine bidimensionnel vertical (Canal numérique) est effectuée. Ce domaine est délimité par un fond horizontal immobile et par une surface libre dont le niveau dépend du temps.

\subsection{Equations fondamentales et conditions aux limites}

On suppose que l'écoulement instationnaire de fluide visqueux est incompressible et bidimensionnel et qu'il est régit par les équations suivantes :

Equation de conservation de la masse

$$
\text { Div } \vec{U}=0
$$

Equation de conservation de la quantité de mouvement

$$
\frac{D \bar{U}}{D t}=-\frac{1}{\rho} \operatorname{grad} P+v \bar{\Delta} \bar{U}+\bar{F}
$$

$\bar{U}(\mathrm{U}, \mathrm{V})$ est la vitesse du fluide; $\mathrm{P}$ est la pression; $\rho$ est la masse volumique ; $v$ est la viscosité cinématique; $\vec{F}$ correspond aux forces extérieures par unité de masse. On ne prendra en compte, ici, que les forces de pesanteur.

Les conditions aux limites appliquées aux frontières du domaine sont :

\subsubsection{Condition aux limites sur le fond du canal}

En raison de la viscosité du fluide, $U=V=0$. Une équation pour la pression est obtenue grâce à la projection verticale de l'équation d'évolution de la quantité de mouvement, et de la condition d'adhérence [3].

\subsubsection{Conditions aux limites à l'entrée du canal numérique (génération de houle)}

Dans un souci de simuler le plus rapidement possible et le plus exactement possible une houle incidente correspondant à la houle réelle, les équations d'entrée du canal numérique sont fondées sur le modèle de houle d'Airy [4]. 


\subsubsection{Conditions aux limites sur la surface libre}

La pression est supposée constante et est prise égale à zéro, et les contraintes tangentielles sont négligées [3].

\subsubsection{Conditions aux limites sur la limite avale}

A la limite aval, on suppose que la houle simulée quitte le domaine sans créer de réflexions [3].

\subsection{Résolution numérique}

Nous avons exploité le système de résolution issu des travaux de Bertelle et al (1991) et Colmard et al. (1996). Une analyse de cette méthode est présentée dans la partie 5 .

Les équations ci-dessus, sont résolues par la méthode des volumes finis. Cette méthode est basée sur la résolution des équations de la mécanique des fluides sous leur forme intégrale. Les relations entre les différentes variables s'expriment sous forme de flux à travers les faces des cellules considérées. Un avantage non négligeable de cette méthode est que le maillage employé peut être déformé verticalement. A partir des équations de conservation de la masse et de quantité de mouvement, il est possible de déterminer une relation pour l'évolution de chaque variable.

Calcul de $U[4]$

$S t \int_{\Omega} \frac{\partial U}{\partial t} d \Omega+\int_{\Omega} \frac{\partial}{\partial x}\left(U^{2}-\frac{v}{\operatorname{Re}} \frac{\partial U}{\partial x}\right) d \Omega+\int_{\Omega} \frac{\partial}{\partial y}\left(U V-\frac{v}{\operatorname{Re}} \frac{\partial U}{\partial y}\right) d \Omega+E u \frac{1}{\rho} \int_{\Omega} \frac{\partial P}{\partial x} d \Omega=0$

avec: Le nombre de Strouhal, $S t=\frac{h}{t \times u}$

Le nombre d'Euler, $E u=\frac{p}{\rho \times u^{2}}$

Le nombre de Reynolds, $\mathrm{Re}=\frac{u \times h}{v}$

et, $\Omega$ : Volume de la cellule à l'instant $t$.

Pour obtenir les valeurs de U, l'équation précédente est mise sous forme d'une matrice tridiagonale. Le système d'équations est résolu par la méthode TDMA [7].

La vitesse verticale est calculée à partir de l'équation de conservation de la masse, l'élévation d'eau est calculée grâce à la condition cinématique, et la pression est calculée grâce à l'équation de Poisson [3]. 
Un mailiage décalé, pour les composantes de vitesses et de pression, est utilisé comme cela se rencontre fréquemment lors des résolutions Eulériennes, dans les méthodes de volumes finis.

On utilise une méthode itérative. A chaque itération, certaines variables apparaissant dans les termes non linéaires seront fixées pour la résolution en cours. Elles seront ensuite actualisées à la fin de l'itération. Un critère de convergence stoppera le processus itératif en assurant que les équations non linéaires soient résolues avec une précision donnée. Notre méthode itérative est construite en calculant successivement les inconnues du problème [8].

A l'instant initial et sur tout le domaine de calcul, les vitesses sont nulles et la répartition des pressions est hydrostatique.

\section{Résultats et étude comparative}

Les conditions de houles sont : La période $\mathrm{T}=1.15 \mathrm{~s}$, la hauteur d'eau $\mathrm{h}=0.27 \mathrm{~m}$ et la hauteur crête-creux : $H=0.038 \mathrm{~m}$ pour le test $\mathrm{H} 2$, et : $\mathrm{H}=0.042 \mathrm{~m}$ pour le test H3.

On s'attend a ce que les résultats du modèle restent différents des valeurs résultants des mesures. Du fait que les fichiers de mesures proviennent d'une estimation avec un certain pourcentage d'erreurs associées aux techniques de mesure, alors que les résultats du modèle sont estimés avec un certain pourcentage d'erreur à cause des approximations mathématique et numériques.

Différents profils de vitesse sont représentés, pour le test H2 (Figure 2), et ce pour différentes distances de l'entrée du canal numérique au bout d'un temps de calcul égal à $\mathrm{t}=10 \mathrm{~T}$.

L'étude comparative [1] a montré les similitudes qualitatives entre les résultats numériques d'une part (figure 2) et les mesures par VDL d'autre part (figure 3). L'étude quantitative pour la phase correspondant au sommet a montré que pour le test $\mathrm{H} 3$, la vitesse horizontale maximale numérique correspondant à l'over flow était localisée au même niveau et avec la même valeur que la valeur expérimentale. En effet, les deux valeurs numériques et expérimentales correspondent à une distance à partir du fond égale à $1.4 \mathrm{~mm}$ [1]

\begin{tabular}{|l|l|l|l|l|l|}
\hline$x(\mathrm{~m})$ & 4 & 4.3 & 4.4 & 4.5 & 4.8 \\
\hline phase $\left(^{\circ}\right)$ & $352^{\circ}$ & $299^{\circ}$ & $275^{\circ}$ & $252^{\circ}$ & $183^{\circ}$ \\
\hline $\mathrm{h}(\mathrm{m})$ & 0.2871 & 0.2763 & 0.2689 & 0.2610 & 0.2497 \\
\hline
\end{tabular}

Tableau 1. Profondeur d'eau, test $\mathrm{H} 2$, résultats numériques. 


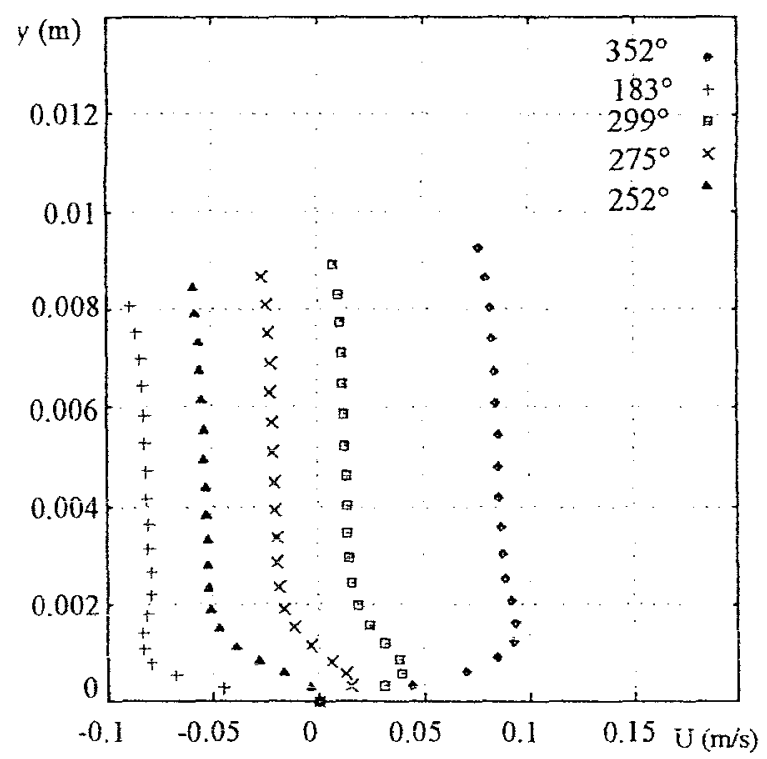

Figure 2. Distribution de vitesse dans la couche limite, Test $\mathrm{H} 2$, résultats numériquies.

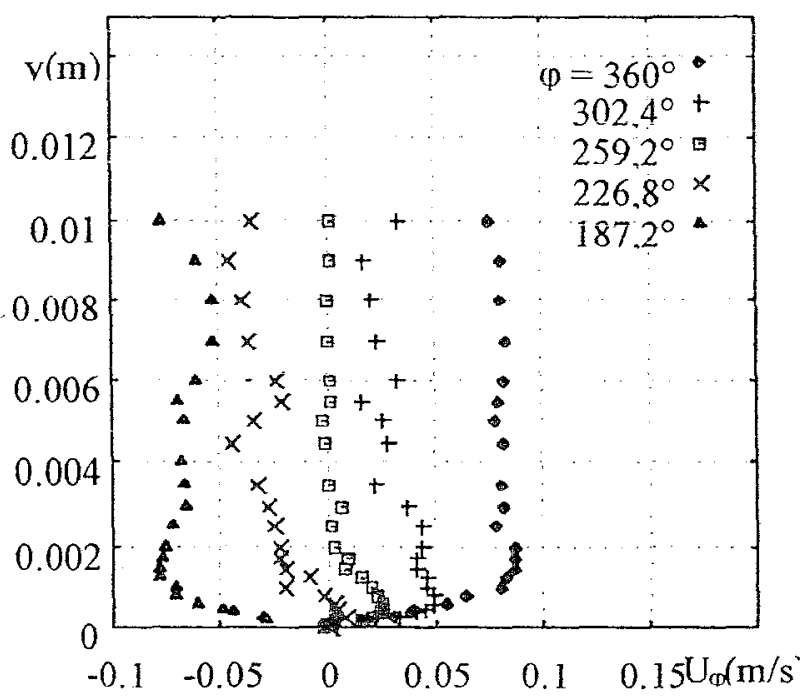

Figure 3. Distribution de vitesse dans la couche limite, Test H2, mesures par VDL [11].

\section{Analyse des résultats}

L'étude comparative a montré de bonnes concordances entres nos résultats numériques et les mesures expérimentales. Cependant une analyse comparative plus fine nécessite d'analyser la méthode de résolution numérique et la technique expérimentale. Une telle analyse est indispensable pour prolonger le modèle au cas ou la couche limite présente un caractère turbulent plus marqué (fond rugueux ou fond de rides). 


\subsection{Analyse de la méthode de résolution}

La première remarque que nous pouvons apporter, concerne la procédure de résolution. En effet, le système n'est pas résolu directement dans sa globalité, l'équation (3) pour les vitesses horizontales étant traitée indépendamment des autres équations. Par ailleurs il convient d'améliorer la discrétisation de l'équation (3) en la traitant globalement, de préférence à une discrétisation terme par terme comme c'est le cas dans cette résolution.

\subsection{Analyse de la technique expérimentale}

Dans cette partie nous analysons les différentes sources de difficultés expérimentales probables. Les éventuelles défaillances au niveau des mesures peuvent être de deux catégories.

La première est la plus probable, elle concerne les possibilités d'une réflexion du faisceau sur le fond due à une concentration accrue de l'énergie lumineuse et ceci pour les mesures près du fond du canal. Cet effet conduit à un éblouissement important et donc à un signal fortement bruité.

La seconde concerne les valeurs de vitesses verticales très près du fond. En effet, pour ces vitesses le défilement des franges est horizontal, et près du fond le réseau de franges d'interférences n'apparaît pas en entier, ce qui ne permet pas à la particule de traverser le nombre de franges nécessaires pour que les mesures soient validées.

\section{Conclusion}

L'étude comparative entre les résuitats numériques et les mesures effectuées sur le canal à houle par V.D.L., a montré que les vitesses au fond pouvaient être calculées correctement par le présent modèle [1]. Nous nous proposons de pouirsuivie cette étude, de manière à modéliser le transport des sédiments par suspension, ce qui nécessite d'introduire l'équation de diffusion appliquée à la concentration dans notre modèle numérique. En parallèle nous envisageons de traiter les équations de Navier-Stokes avec surface libre en utilisant le concept de pseudo-compressibilité. Pour ne pas altérer le caractère conservatif associé aux variables $u, v$ et $p$, nous traitons la surface libre par un concept de type "Level set" [9]. En ce qui concerne la partie expérimentale, notre idée consiste à peindre le fond du canal en noir, ceci permettra pour les prochaines mesures de réduire considérablement le problème de réflexion du laser, d'où un signal peut bruité. 


\section{Références}

[1] Absi, R., Abdelgader, A., and Bélorgey, M., 1997. Bottom Boundary Layer Study for the Sediment Transport Modeling. Proc. 3rd Int. Conf. Mediterranean Coastal Environment. MEDCOAST 97, Qawra, Malta, E. Özhan (Ed.). pp. 12751285.

[2] Bélorgey, M., Le Bas, J., and Grandjean, A., 1989. Application of Laser Doppler Velocimetry to the Study of Turbulence Generated by Swell in the Vicinity of Walls or Obstacles. Coastal Engineering, vol.13, pp. 746-765.

[3] Bertelle, C., and Lhuissier, J. F., 1991. Simulation numérique d'un canal a houle. Troisiémes journées de l'hydraudynamique, IMG Grenoble, pp. 149-161.

[4] Colmard, C., Bélorgey, M., and Aupérin, L., 1996. Hydraulic Functioning of "Fixed Water Wall" Type Breakwaters. Proc. 6th Int. Offshore Polar Engineering Conf., Los Angeles, USA, pp. 531-537.

[5] Durst, F., Melling, A., and Whitelaw J.H., 1981. A Principles and Practice of Laser Doppler Anemometry. Academic press.

[6] Fredsoe, J., and Deigaard, R., 1992. Mechanics of Coastal Sediment Transport. Advanced Series on Engineering - Volume 3, World Scientific Publishing.

[7] Patankar, S. V., 1980. Numerical Heat Transfer and Fluid Flow. Hemisphere Publishing corporation.

[8] Sarraf, 1987. Three Dimensional Surface Water Modelling Using a Mesh Adaptive Technique. Int. J. for Num. Meth. in Fluids, Vol. 7, pp. 465-488.

[9] Sussman, M., Smereka, P., and Osher, S., 1994. A Level Set Approch for Computing Solutions to Incompressible Two-Phase Flow. Journal of Computational Physics, Vol. 114, pp. 146-159.

[10] Savioli, J., and Justesen, P., 1997. Sediment in oscillatory flows over a plane bed. Journal of Hydraulic Research, Vol. 35, No. 2, pp. 177-I89.

[11] Tcheukam-Toko, D., 1997. Etude de la couche limite générée par l'action de la houle et/ou du courant sur fond hydrauliquement lisse : Application au transport sédimentaire. Thèse de Doctorat, Université du Havre. 